\section{A guide to good behavior}

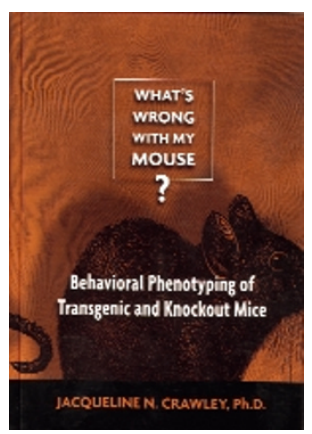

What's Wrong with my Mouse?

by Jacqueline $N$. Crawley

John Wiley \& Sons, New York, 2000. \$79.95

hardcover, pp 352

ISBN 0-471-31639-3

\section{Reviewed by Robert Gerlai}

A decade ago, the mere production of a transgenic or knockout mouse could make the news, and the authors of such studies were often rewarded by papers in Science or Nature. Nowadays, transgenic mice can be custom-ordered from biotech companies that generate hundreds of mutants a year, and their production is becoming ever easier, as newer and more efficient technologies are developed. But what do we do with all these mutants?

Jacqueline Crawley offers some answers in her new book, What's Wrong with my Mouse? Given that at least 30\% of mammalian genes are expressed in the brain, there is a tremendous need for behavioral and neurobiological analysis of mutant mice. Crawley aims to introduce the novice to the wide variety of behavioral tests that are now available, and has succeeded in providing a timely and useful overview of the field.

The book is written primarily for the non-behavioral scientist. Crawley explains that "behavior is not a cookbook discipline," and the book is not intended to give detailed recipes. Rather, her aim is to introduce molecular biologists and neuroscientists to some of the most frequently used behavioral paradigms. Behavioral analysis is often seen as a 'soft' science, in contrast to the supposed sophistication and rigor of molecular genetics, but in fact the problem often lies with the choice and execution of procedures. It is deceptively easy to generate quantitative data from behavioral studies, and the field presents many pitfalls for the unwary. Crawley's book should help inexperienced researchers to avoid many common errors, and it also provides valuable pointers for establishing collaborations with colleagues

Robert Gerlai is at the Neuroscience Department, Lilly Research Laboratories,

Lilly Corporate Center, Drop Code 0510,

Indianapolis, Indiana 46285, USA.

e-mail:gerlai_robert@lilly.com who have the necessary expertise.

The least satisfactory part of the book is the second chapter, which provides an overview of the basics of transgenic and knockout technologies. Many of the book's target readers will already be familiar with this area, and will find Crawley's treatment weak. There are a number of incorrect statements or confusing remarks here. For instance, homologous recombination is not a random event, as implied on page 10 . Current technology does not attempt to define the integration site of transgenes using tissue-specific promoters (page 11), but rather attempts to direct expression of the transgene in a tissue-specific manner with the help of such promoters. Coat color of chimeras is not a marker of a successful mutation (page 14), but rather of successful incorporation of ES cells into the chimeric animal. Random segregation events do not create unusual alleles as stated on page 21. Transgenic technology is not Crawley's main expertise, and readers seeking an introduction to this field would be well advised to skip this chapter and turn elsewhere, for instance to the sources listed at the end of the chapter.

The remainder of the book is much better. Chapter 3 discusses the importance of analysis of general health. Scientists focusing on the 'function' of their favorite gene often forget that behavioral phenotypes may not always reflect direct effects on the central nervous system-mutations can affect numerous other organs in ways that can significantly influence the outcome of a behavioral study. A thorough check of the animals' general health is therefore an essential part of any phenotypic characterization, and this section provides numerous simple examples of what to measure and how to conduct the analysis. Chapters 4 and 5 are on motor and sensory function, and will be useful to scientists interested in these systems. Researchers studying other aspects of behavior will also find many useful suggestions here: motor and sensory deficits can be important confounds, for example, when studying higher cognitive functions. Chapter 6 presents some of the basic tests designed to analyze different forms and aspects of learning and memory. These include the famous 'water maze' task, along with classical operant conditioning and tasks that test attention or motor learning. This section also provides a good treatment of more modern and ethological approaches to memory, including tests of social recognition and socially transmitted food preference. The following chapters (7-11) cover a broad range of behavioral phenomena, including feeding and drinking, reproductive and social behaviors, emotional behaviors and reward. In addition to presenting several of the more established tests, each of these chapters ends with examples from the mouse transgenic literature.

Crawley's style is engaging and thoughtprovoking, and the text is punctuated by interesting metaphors and jokes. It reads easily, and the overall impression is of a helpful guide taking the reader step by step through the complexities of animal behavioral research. The book is also a good source of bibliographic information, with suggested readings and other practical information at the end of each chapter.

Experienced behavioral neuroscientists may find some of the topics treated superficially and may also spot occasional errors in the methods, results and citations. This is probably unavoidable, given the vastness of the literature and the wide range of topics covered. Another weakness of the book is the way results from transgenic and gene targeting studies are presented: although the idea of discussing such examples at the end of each behavioral chapter is a good one, the text is too condensed and often seems to be a random list of facts and findings. Nevertheless, the quantity of information gathered together in this work itself makes it a useful read.

The need for such a book is clear. Now that the production of mutants is so efficient, the bottleneck in neurobehavioral genetic research is usually the phenotypic characterization of the mutants. The US National Institutes of Health, among other agencies, has recognized this, and has recently initiated several funding programs for the development and use of behavioral phenotyping of mutant mice as well as another model organism, zebrafish. Crawley's book is thus very timely and will enable the non-behavioral scientist to make informed decisions on what behavioral tests are needed and with whom they should collaborate. 